\title{
Saturation of relaxational absorption in amorphous metals at very high acoustic powers
}

\author{
W. Arnold (*), P. Doussineau (**) and A. Levelut (**) \\ (*) Fraunhofer-Institute for Non-Destructive Testing, Bldg 37, University, 6600 Saarbruecken, \\ F.R.G. \\ ${ }^{(* *)}$ Laboratoire d'Ultrasons (***), Université Pierre et Marie Curie, Tour 13, 4, place Jussieu, \\ 75230 Paris Cedex 05, France
}

(Reçu le 14 mai 1982, révisé le 2 août, accepté le 19 août 1982)

\begin{abstract}
Résumé. - Des effets non-linéaires récemment observés sur l'absorption et la dispersion acoustiques dans $\mathrm{PdSiCu}$ amorphe et dans d'autres métaux amorphes sont expliqués dans le cadre de la théorie des systèmes à deux niveaux (S2N). L'origine supposée de ces effets est l'égalisation des populations des deux niveaux des S2N par l'onde acoustique sur une largeur spectrale pouvant atteindre $k T / \hbar$, ce qui entraîne une saturation de l'atténuation de relaxation.

Abstract. - Strong nonlinear effects recently observed in the acoustic absorption and dispersion in amorphous $\mathrm{PdSiCu}$ and other amorphous metals are explained within the framework of the two-level system (TLS) theory. It is proposed that these nonlinear effects originate from equalization of the TLS occupation numbers by the acoustic wave over a spectral width of the order of $k T / \hbar$, causing saturation of the relaxation absorption.
\end{abstract}

Amorphous metals as well as insulating glasses contain low-energy excitations generally called two-level systems (TLS) and which determine to a large extent their low temperature properties [1]. Quite characteristic is their acoustic behaviour. At very low temperatures $(T \lesssim 1 \mathrm{~K})$ the sound velocity first increases logarithmically with temperature, then passes through a maximum and thereafter decreases. In the same temperature range, the absorption increases rapidly with temperature in amorphous insulators (as $T^{3}$ ) and less steeply in metals (as $T$ ) and is frequency independent. With increasing temperature this absorption then becomes temperature-

(***) Associated with the Centre National de la Recherche Scientifique. 
independent, as has been indeed observed in various amorphous materials [2, 3, 4]. Such an absorption originates from relaxation effects caused by strain modulation of the energy levels of the TLS, and the relaxation rate reflects the coupling of the TLS to phonons and/or electrons [5, 6]. Furthermore, the ultrasonic absorption is dependent on the acoustic intensity. At low intensities, this well-established effect is due to the saturation of the resonant absorption by two-level systems. The intensity at which saturation becomes noticeable depends on the relaxation rate of the TLS, which in turn allows one to obtain information on the origin of the relaxation mechanism [1]. Until now it was believed that the relaxation absorption due to TLS is power independent and that the only nonlinear absorption mechanism is saturation of the resonant process.

Recently, a series of papers have appeared presenting unexpected experimental results on amorphous PdSiCu [7, 8], PdSi [9], and PdNiP [10]. These investigations revealed a saturation effect of the ultrasonic absorption at power levels far higher than previously observed [4]. Furthermore, the absolute value of the unsaturated absorption was much higher than expected for a resonant absorption process, and the measured temperature and frequency dependence of the absorption was not in line with predictions of the theory [11,12] and, in fact, seemed to be in severe contradiction with other experimental work [4]. This effect has also been found in polycrystalline $\mathrm{NbZr}$ containing low-energy excitations [13]. A more recent paper at least removes the contradictions between the various experimental results by showing that two different saturation stages must exist in these materials [14] : at a moderate acoustic flux a first saturation effect appears which is obviously the one already known (stage I) and which can be written as [12] :

$$
\begin{aligned}
& \alpha_{\mathrm{I}}=\alpha_{0}\left(1+\Phi / \Phi_{\mathrm{c}}\right)^{-1 / 2} \\
& \alpha_{0}=\frac{\pi \omega \bar{P} \gamma^{2}}{\rho v^{3}} \tanh (\hbar \omega / 2 k T) \simeq \frac{\pi \hbar \bar{P} \gamma^{2}}{2 \rho v^{3}} \frac{\omega^{2}}{k T}
\end{aligned}
$$

Here, $\Phi$ is the acoustic flux, $\Phi_{\mathrm{c}}$ is the critical flux, $\bar{P}$ is the density of states of the TLS, $\gamma$ is their coupling coefficient to phonons, $\rho$ is the density, $v$ is the sound velocity, and $\omega / 2 \pi$ is the frequency of the sound wave. For higher values of acoustic power ( $20 \mathrm{~dB}$ higher) a second saturation effect becomes noticeable (stage II), and the following empirical formula was found to describe this absorption mechanism quite well :

$$
\alpha_{\mathrm{II}}=\alpha_{0}^{\prime}\left(1+\Phi / \Phi_{\mathrm{c}}^{\prime}\right)^{-1 / 2}
$$

$\alpha_{0}^{\prime}$ was found to increase linearly with the frequency and to be temperature independent below about $0.6 \mathrm{~K}$. Above $0.6 \mathrm{~K} \alpha_{0}^{\prime}$ decreases when the temperature increases. Equation (2) also holds for the previously published results $[7,8,9,10]$. It is startling that this effect apparently also displays the familiar power dependence of resonant absorption due to TLS.

The purpose of the present letter is to propose an explanation for these new experimental results. We think that it is possible to fully understand them in terms of the already very well developed dynamics of TLS in glassy systems. A strong acoustic wave saturates the TLS population within a bandwidth related to the transverse relaxation time $T_{2}$. For a distribution of level splittings $\hbar \omega_{0}$, as it is the case in glasses, this bandwidth increases with increasing power [15] :

$$
\Gamma_{2}^{\prime}=\Gamma_{2} \sqrt{1+\Phi / \Phi_{c}}
$$

where $\Gamma_{2}=T_{2}^{-1}$ is the bandwidth in the limit $\Phi=0$. As a first result the transverse susceptibility $\chi_{\perp}$ of the TLS is modified, and saturation of the acoustic absorption as described by equation (1) follows straightforwardly by taking into account the distribution of level splittings of the 
TLS. $\Gamma_{2}$ and $\Gamma_{1}$ (which is the inverse of the longitudinal relaxation time $T_{1}$ ) enter the expression of the critical flux : $\Phi_{\mathrm{c}}$ is proportional to $\Gamma_{1} \Gamma_{2}[11,12]$.

We propose that there is also an effect on the longitudinal susceptibility $\chi_{\|}$which governs the relaxational sound attenuation and dispersion. The relaxation absorption is dominated by TLS with splittings $\hbar \omega_{0}$ up to a few times $k T$. The acoustic wave saturates the TLS populations, reducing $\chi_{\|}$over an energy range $\hbar \Gamma_{2}^{\prime}$. As long as $\hbar \Gamma_{2}^{\prime} \ll k T$ this effect is negligible because the hole burnt in the population is too narrow. However, at very high acoustic flux, such that $\hbar \Gamma_{2}^{\prime} \gtrsim k T$, all the TLS normally efficient in the relaxation process have their longitudinal susceptibility $\chi_{\|}$ strongly reduced, and the corresponding acoustic attenuation goes to zero.

More precisely, our explanation of the experimental results is based on the following hypotheses :

(i) The effective coupling constant $C=\bar{P} \gamma^{2} / \rho v^{2}$ for the resonant absorption is also valid for relaxation absorption. This implies that the distribution function of TLS is the same as originally proposed [16].

(ii) In amorphous metals, the transverse relaxation time $T_{2}$ of the TLS is very short, and apparently of the order of $T_{1}$ [17], and therefore one can write [18] :

$$
T_{2}=2 T_{1} \text {. }
$$

These short relaxation times originate from the strong coupling of TLS to electrons [6].

(iii) We assume for the longitudinal susceptibility :

$$
\chi_{\|}\left(\omega, \omega_{0}, \Phi\right)=\chi_{\|}\left(0, \omega_{0}, 0\right) \frac{1}{1+i \omega T_{1}} \frac{\omega_{0}^{2}+\Gamma_{2}^{2}}{\omega_{0}^{2}+\Gamma_{2}^{2}\left(1+\Phi / \Phi_{c}\right)} .
$$

The third factor in this equation describes the change of population difference of the TLS due to the acoustic flux at frequencies $\omega / 2 \pi$. We admit that the form of equation (5) is difficult to justify because the exact calculation of the nonlinear behaviour of a spin ensemble is probably not possible if the saturating field is not circularly polarized and when the condition $\omega T_{2} \gg 1$ does not apply.

(iv) We assume that the total attenuation is simply the sum of the relaxation attenuation (which is now power-dependent) and the resonant attenuation. This point is not obvious, since both mechanisms are nonlinear.

(v) Thermal phonons do not contribute appreciably to the relaxation rate of the TLS in the materials under consideration. This is certainly true at least up to $\sim 3 \mathrm{~K}$. In this case the inverse longitudinal relaxation time is given by $T_{1}^{-1}=r K_{1} \frac{\hbar \omega_{0}}{2 k} \operatorname{cotanh}\left(\frac{\hbar \omega_{0}}{2 k T}\right)$, where the constant $K_{1}$ is a measure of the TLS-electron coupling [6] and $r$ is a parameter which describes the distribution of the coupling strength of the TLS. The minimum relaxation time $T_{1}^{\mathrm{m}}$, which corresponds to the strongest coupling, is obtained with $r=1$.

The relaxational absorption is given by

$$
\alpha_{\text {rel }}=\frac{2 \omega C}{v} \int \mathrm{d} \omega_{0} \int \mathrm{d} r \frac{\sqrt{1-r}}{r} \operatorname{Im} \chi_{\|}\left(\omega, \omega_{0}, \Phi\right) .
$$

We now insert equation (5) into equation (6) using

$$
\chi_{\|}\left(0, \omega_{0}, 0\right)=-\frac{\hbar}{4 k T} \operatorname{sech}^{2}\left(\frac{\hbar \omega_{0}}{2 k T}\right) .
$$

In equation (5) the integral over $r$ was done analytically while the integral over $\omega_{0}$ was calculated numerically. 


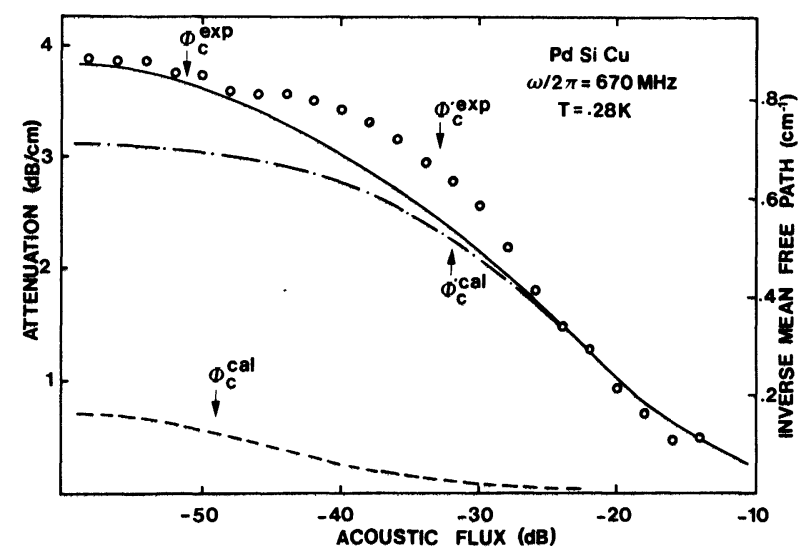

Fig. 1. - Power-dependent absorption in the amorphous metal PdSiCu for a frequency of $670 \mathrm{MHz}$ and a temperature of $0.28 \mathrm{~K}$. Theoretical results are represented by the solid line, and experimental points by the open circles [14]. The theoretical calculation is the sum of the resonant absorption (dashed line) and the power-dependent relaxation absorption (dashed-dotted line) obtained using $v_{T}=1.8 \times 10^{5} \mathrm{~cm} / \mathrm{s}$, $C=4.1 \times 10^{-5}$ and $K_{1}=2 \times 10^{10} \mathrm{~K}^{-1} \mathrm{~s}^{-1} . \Phi_{\mathrm{c}}^{\exp }$ and $\Phi_{\mathrm{c}}^{\prime \exp }$ are experimental values from reference [14]. $\Phi_{\mathrm{c}}^{\mathrm{cal}}$ and $\Phi_{\mathrm{c}}^{\text {cal }}$ are the values calculated as explained in the text.

In figure 1 we show the result for an ultrasonic frequency of $670 \mathrm{MHz}$ and a temperature of $0.28 \mathrm{~K}$ (solid line) together with the corresponding experimental results (open circles) for $\mathrm{PdSiCu}$ [14]. With increasing acoustic power the absorption indeed decreases. Using $v_{T}=1.8 \times 10^{5} \mathrm{~cm} / \mathrm{s}$ we obtain the best fit with the following parameters : $K_{1}=2.0 \times 10^{10} \mathrm{~K}^{-1} \mathrm{~s}^{-1}$, $C=4.1 \times 10^{-5}$. They are very close to those obtained from previous experimental results on the same material [4]. The agreement between theory and experiment is quite satisfactory, although there is a slight discrepancy, as can be seen in figure 1 . We think that this is of minor importance considering the stage of the present theory and also the experimental data available. However, we should like to point out the following :

a) Equation (5) is only valid in the present form, if there is a sufficiently large thermal reservoir in order to define a temperature $T$ at which the sample is held. Saturation of the whole ensemble of TLS up to $k T$ is equivalent to increasing the temperature of the TLS. In the case of the experiments in the non-superconducting metallic glasses $[7,8,9,10,14]$ the thermal reservoir is provided by the electrons since their specific heat is at least two orders of magnitude larger than the specific heat caused by the TLS.

b) In the case of $\mathrm{NbZr}$ [13] there arises a problem. At $T \sim 0.5 \mathrm{~K}$, where the nonlinear effects are observed, the specific heat is mostly due to TLS $\left(T_{c} \sim 8.6 \mathrm{~K}\right)$, and consequently the temperature $T$ of the sample should be elevated if our physical idea is correct. One therefore should rewrite equation (5) taking this into account. In the real experiments, however, the sample always has to be mounted to some cooling bath which might quite easily provide the additional reservoir.

Power-dependent relaxational absorption raises questions about whether there exists a critical intensity $\Phi_{c}^{\prime}$, as it is the case in resonant absorption (Eq. (1)), and on which parameters $\Phi_{c}^{\prime}$ may depend. We have not derived an analytical expression for $\Phi_{c}^{\prime}(\omega, T)$, but we can at least define $\Phi_{\mathrm{c}}^{\prime}$ by

$$
\alpha_{\text {rel }}\left(\Phi_{\mathrm{c}}^{\prime}\right)=\alpha_{\mathrm{rel}}(0) / \sqrt{2}
$$

which corresponds to the experimental findings of equation (2). 


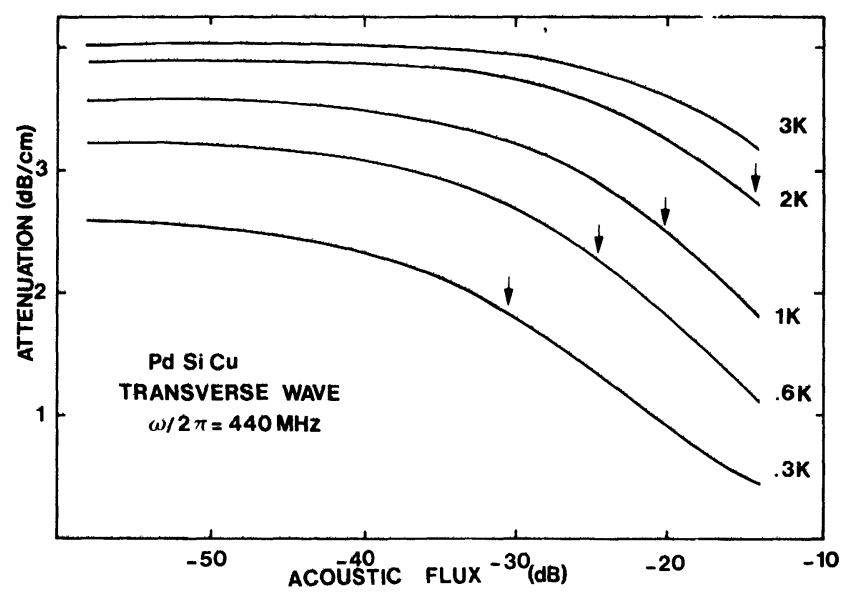

Fig. 2. - Power-dependent relaxation absorption at various temperatures for the ultrasonic frequency $\omega / 2 \pi=440 \mathrm{MHz}$. The calculations hold for parameters appropriate for the amorphous metal PdSiCu and cover the same power range as that used in the experiments [14]. The critical flux (indicated by the arrows) as defined by equation (8) increases with increasing temperature in agreement with the experiment.

We now focus our attention on the comparison between theory and experimental results at higher temperatures. Our calculations yield a saturable relaxation attenuation which increases as $T$ increases and which tends to a constant value when the condition $\omega T_{1}^{\mathbf{m}} \lesssim 1$ is fulfilled. The constant value corresponds to the plateau [5] observed at higher temperatures in various materials. The interpretation given in [14] of the experimental data leads to the conclusion that the attenuation is constant up to $0.6 \mathrm{~K}$ and then decreases with temperature. We believe that this is an artefact of the analysis of data obtained over only a limited range of acoustic flux. We also find that the intensity at which saturation sets in becomes larger with higher temperatures, which is in line with the experimental data [14]. However, we should like to point out that it is not possible to infinitely increase the intensity in the experiment. As a consequence, the higher the temperature, the more difficult it is to fully saturate the relaxation absorption, and hence it becomes increasingly inaccurate to deduce the unsaturated absorption $\alpha_{\mathrm{rel}}$. Figure 2 shows the result of a series

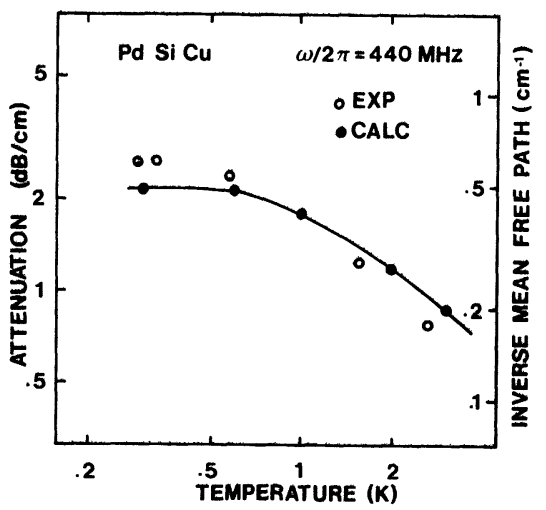

Fig. 3. - Saturable absorption $\alpha_{\text {rel }}$ calculated for the five temperatures as indicated in figure 2 (full circles) compared with experimental results (open circles) [14]. The solid line through the calculated points is a guide for the eye. 


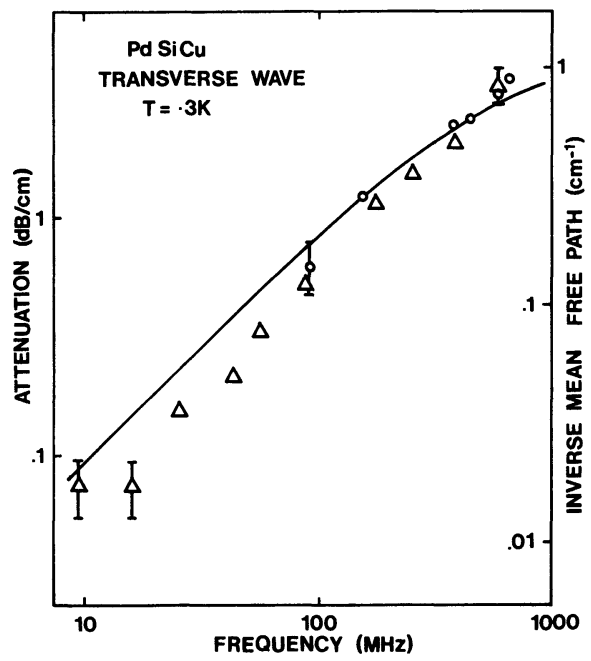

Fig. 4. - Frequency dependence of the relaxational absorption at vanishing power levels at a temperature of $0.3 \mathrm{~K}$ (solid line) compared with experimental results (open circles from [14], triangles from [8]). The two symbols refer to different samples.

of numerical calculations for the acoustic frequency $\omega / 2 \pi=440 \mathrm{MHz}$ at various temperatures. Let us assume that the relaxational saturated attenuation is the difference $\Delta \alpha$ between the attenuation at very low acoustic flux and the attenuation at the highest flux in [14]. Using this assumption we can obtain this quantity from figure 2 . The result is displayed in figure 3 , together with the experimental data [14]. Surprisingly, the fit is quite reasonable if we consider the approximations used in the theory and the arbitrariness of our procedure.

Finally we show in figure 4 the frequency dependence of our calculations for $T=0.3 \mathrm{~K}$ together with corresponding experimental data $[8,14]$. Our results are quite trivial. Because the temperature is low the saturated attenuation $\Delta \alpha$ is practically equal to $\alpha_{\text {rel }}(\omega, T)$, and the linear dependence of $\Delta \alpha$ on $\omega$ is very well established as long as $\omega T_{1}^{\mathrm{m}} \lesssim 1$.

There have also been efforts to observe the effect considered here in fused silica but they failed [7] and we think that the reason is straightforward : the relaxation absorption in $\mathrm{a}-\mathrm{SiO}_{2}$ at $T=0.3 \mathrm{~K}$ is of the order of $0.06 \mathrm{~dB} / \mathrm{cm}$ which is hardly observable.

We should like to mention that according to our theory the sound velocity caused by relaxation becomes also saturable. However, a complete treatment of all relevant effects is not straightforward, because the resonant contribution to the dispersion may also become power dependent, as has been shown experimentally [17] and theoretically [20]. We shall present corresponding calculations in a later paper.

In summary, we have proposed an explanation for the recently discovered nonlinear acoustic absorption in amorphous metals. Our explanation is entirely based on the dynamics of the TLS. We should like to emphasize that our interpretation of the experimental data is a good illustration of the power of the theory of TLS inherent in amorphous materials.

Acknowledgments. - We thank Prof. Elbaum for communicating to us the experimental results of his group prior to publication, and we also thank Prof. Jäckle for helpful comments. 


\section{References}

[1] For recent review see collected papers in Amorphous Solids, edited by W. A. Phillips (Springer Verlag) 1981.

[2] Doussineau, P., Frénois, C., Leisure, R. G., Levelut, A. and Prieur, J.-Y., J. Physique 41 (1980) 1193.

[3] Doussineau, P. and Robin, A., in Phonon Scattering in Solids, edited by H. J. Maris (Plenum Press) 1980, p. 65.

[4] Doussineau, P., J. Physique-Lett. 42 (1981) L-83.

[5] JäCKLE, J., Z. Phys. 257 (1972) 212.

[6] Golding, B., Graebner, J. E., Kane, A. B. and Black, J. L., Phys. Rev. Lett. 41 (1978) 1487.

[7] Araki, H., Park, G., Hikata, A. and Elbaum, C., Solid State Commun. 32 (1979) 625 ; Phys. Rev. B 21 (1980) 4470.

[8] Park, G., Hikata, A. and Elbaum, C., Phys. Rev. B 23 (1981) 5597.

[9] Park, G., Hikata, A. and Elbaum, C., J. Non-Cryst. Solids 45 (1981) 93.

[10] Park, G., Hikata, A. and Elbaum, C., Phys. Rev. B 24 (1981) 7389.

[11] Jofrrin, J. and Levelut, A., J. Physique 36 (1975) 811.

[12] Hunklinger, S. and Arnold, W., in Physical Acoustics, edited by W. P. Mason and R. N. Thurston (Academic Press) 1976, Vol. 12, p. 156.

[13] Wang, J. L., Weiss, G. and Hunklinger, S., Proc. 5th Int. Conf. «Physics of Non Crystalline Solids ", to be published in J. Physique Colloque 43 (1982).

[14] Hikata, A., Cibuzar, G. and Elbaum, C., J. Low Temp. Phys. 49 (1982) № 3-4 (to appear).

[15] Allen, E. G. L. and Eberly, J. H., Optical resonances and two-level Atoms (J. Wiley, New York) 1975, p. 142.

[16] Phillips, W. A., J. Low Temp. Phys. 7 (1972) 351.

[17] Cordié, P. and Bellessa, G., Phys. Rev. Lett. 47 (1981) 106.

[18] Bloembergen, N., Phys. Rev. 78 (1950) 572.

[19] Doussineau, P., Legros, P., Levelut, A. and Robin, A., J. Physique-Lett. 39 (1978) L-285.

[20] Continentino, M. A., Solid State Commun. 40 (1981) 781. 